\title{
Antiresonant Hollow Core Fiber with Octave Spanning Bandwidth for Short Haul Data Communications
}

\author{
J.R. Hayes*, S.R. Sandoghchi, T.D. Bradley, Z. Liu, R. Slavík, M.A. Gouveia, N.V. Wheeler, G. Jasion, \\ Y. Chen, E. Numkam Fokoua, M.N. Petrovich, D.J. Richardson and F. Poletti \\ Optoelectronics Research Centre, University of Southampton, Highfield, Southampton, SO17 1BJ, United Kingdom \\ Authore-mail address: jrh@orc.soton.ac.uk
}

\begin{abstract}
We report an effectively singlemode tubular antiresonant hollow core fiber with $>1000 \mathrm{~nm}$ bandwidth and record-low loss of $25 \mathrm{~dB} / \mathrm{km}$, fully connectorizable with SMF28. We demonstrate penalty-free data transmission at wavelengths of 1065,1565 and $1963 \mathrm{~nm}$. OCIS codes: (060.4005) Microstructured fiber; (060.0060) Fiber optics and optical communications.
\end{abstract}

\section{Introduction}

Hollow core fibers based on photonic bandgap guidance (HC-PBGFs) have proven themselves an attractive technology for low latency data transmission [1], with experiments over length scales of practical relevance ranging from a few [2] to a few tens of $\mathrm{km} \mathrm{[3]} \mathrm{so} \mathrm{far} \mathrm{demonstrated.} \mathrm{The} \mathrm{bandwidth} \mathrm{of} \mathrm{the} \mathrm{single} \mathrm{low-loss} \mathrm{operating} \mathrm{window}$ for HC-PBGFs currently ranges from $\sim 20 \mathrm{~nm}$ for the lowest loss fiber reported to date [4], to $200 \mathrm{~nm}[2,5]$ for fibers of slightly higher loss, with the operating bandwidth typically centered either on the C-band or at their wavelength of predicted minimum loss, around $2 \mu \mathrm{m}$. While very high data capacity can be achieved in these fibers using dense WDM schemes [6], cheaper ultra-coarse WDM transmission and/or additional capacity would benefit from an increased bandwidth. A significantly broader bandwidth can be achieved in air-core antiresonant fibers (ARFs) where guidance is based on antiresonance from thin glass membranes surrounding the hollow core, and the cladding consists of either a Kagome-type lattice [7], or a single ring of touching [8] or non-touching tubes [9,10]. However, ARFs reported to date typically exhibit a structured transmission spectrum, with multiple loss peaks arising from resonant coupling between air and glass modes located at nodes in the cladding. These resonances are difficult to control and give rise both to higher loss $(\sim 50-300 \mathrm{~dB} / \mathrm{km}$ typical) and poorly reproducible modal properties. Consequently, to date no data transmission experiments have ever been reported in any form of ARF.

In this work we demonstrate for the first time that ARF technology has the potential to make an impact on short reach data transmission. We present a non-contacting tubular fiber, comprising a single ring of 7 tubes around a hollow core (Fig.1a), with the lowest loss for this fiber type $(\sim 25 \mathrm{~dB} / \mathrm{km})$ and the widest sub-100 dB/km transmission window for any air-guiding fiber ever reported $(>1100 \mathrm{~nm}$ or $>1$ octave). Due to its high differential modal loss and low intermodal coupling, an excellent modal purity ( $>50 \mathrm{~dB}$ suppression of high order modes) can be achieved after just a few 10 s of meters of fiber. This, combined with a spectrally smooth loss curve, allows lowlatency data transmission over an unprecedentedly broad wavelength range. To demonstrate this, we report penalty free $10 \mathrm{Gbit} / \mathrm{s}$ data transmission at wavelengths of $1065 \mathrm{~nm}, 1556 \mathrm{~nm}$ and $1963 \mathrm{~nm}$ in the same $100 \mathrm{~m}$ long fiber.
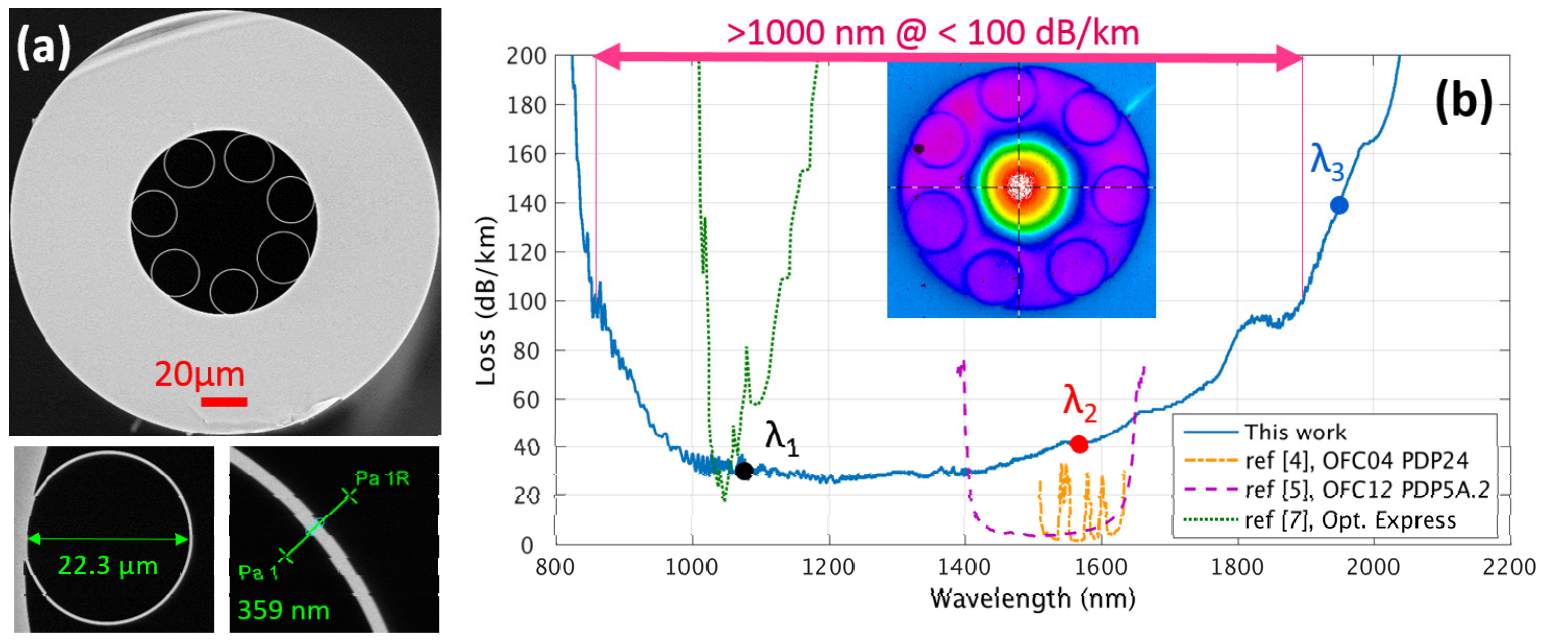

Fig.1. Fabricated hollow core tubular fiber: (a) SEM images of the full fiber cross section and details of the cladding; (b) Measured loss spectrum (blue curve) compared with state-of-the-art hollow core PBGFs (orange/purple) and Kagome ARF (green) highlighting the exceptional bandwidth and spectral flatness of the present fiber. Inset: measured mode image after 5 meters. 


\section{Fiber fabrication and characterization}

The fiber was produced using a two stage stack-and-draw process. A key fabrication step was the manufacture of uniform capillaries $(4.09 \pm 0.02 \mathrm{~mm}$ in diameter, with $0.24 \mathrm{~mm}$ thick walls) used to form the cladding tubes, which requires excellent uniformity. During the second draw stage pressure control was used to allow the capillaries to expand and to achieve a final aspect ratio (inner to outer diameter ratio) of $\sim 0.97$. The fiber draw parameters were slightly tuned during the draw to produce fibers centered at different wavelengths. The current process yield is $\sim 2.1$ $\mathrm{km}$ but we envisage it could be straightforwardly scaled up to multi-km. Loss measurements were achieved via a cutback method employing a supercontinuum source, two optical spectrum analyzers (400-1750 nm and 1200-2400 $\mathrm{nm}, 2 \mathrm{~nm}$ resolution) to cover the full fiber bandwidth, and the input end mode-matched and spliced to a standard single mode fiber (SMF). The minimum measured loss was $16 \mathrm{~dB} / \mathrm{km}$ near $820 \mathrm{~nm}$ in a $38 \mu \mathrm{m}$ core fiber with a transmission extending from 820 to $1800 \mathrm{~nm}$. A slightly different fiber had a minimum loss of $\sim 25 \mathrm{~dB} / \mathrm{km}$ and $<120$ $\mathrm{dB} / \mathrm{km}$ for all wavelengths between $900 \mathrm{~nm}$ and $2050 \mathrm{~nm}$. Figure 1 shows details of a third fiber, 100 meters long, that we have selected for more detailed analysis. It has a core diameter of $40.2 \mu \mathrm{m}$, an average tube diameter of 22.6 $\mu \mathrm{m}$ (with a standard deviation of $0.9 \mu \mathrm{m}$ ) and an average membrane thickness of $\sim 360 \mathrm{~nm}$ (Fig. 1a). Its cutback loss is compared in Fig.1b to the lowest reported loss HC-PBGF [4] and ARF [7], and to a wide bandwidth HC-PBGF [5], to highlight the exceptionally wide $(>1000 \mathrm{~nm})$ air-guiding bandwidth of this fiber. Unlike other ARFs reported in the literature the loss is spectrally very smooth and extremely flat in the $400 \mathrm{~nm}$ wide region around its minimum loss wavelength. Fig. 2 shows other measured and simulated optical properties of the fiber.
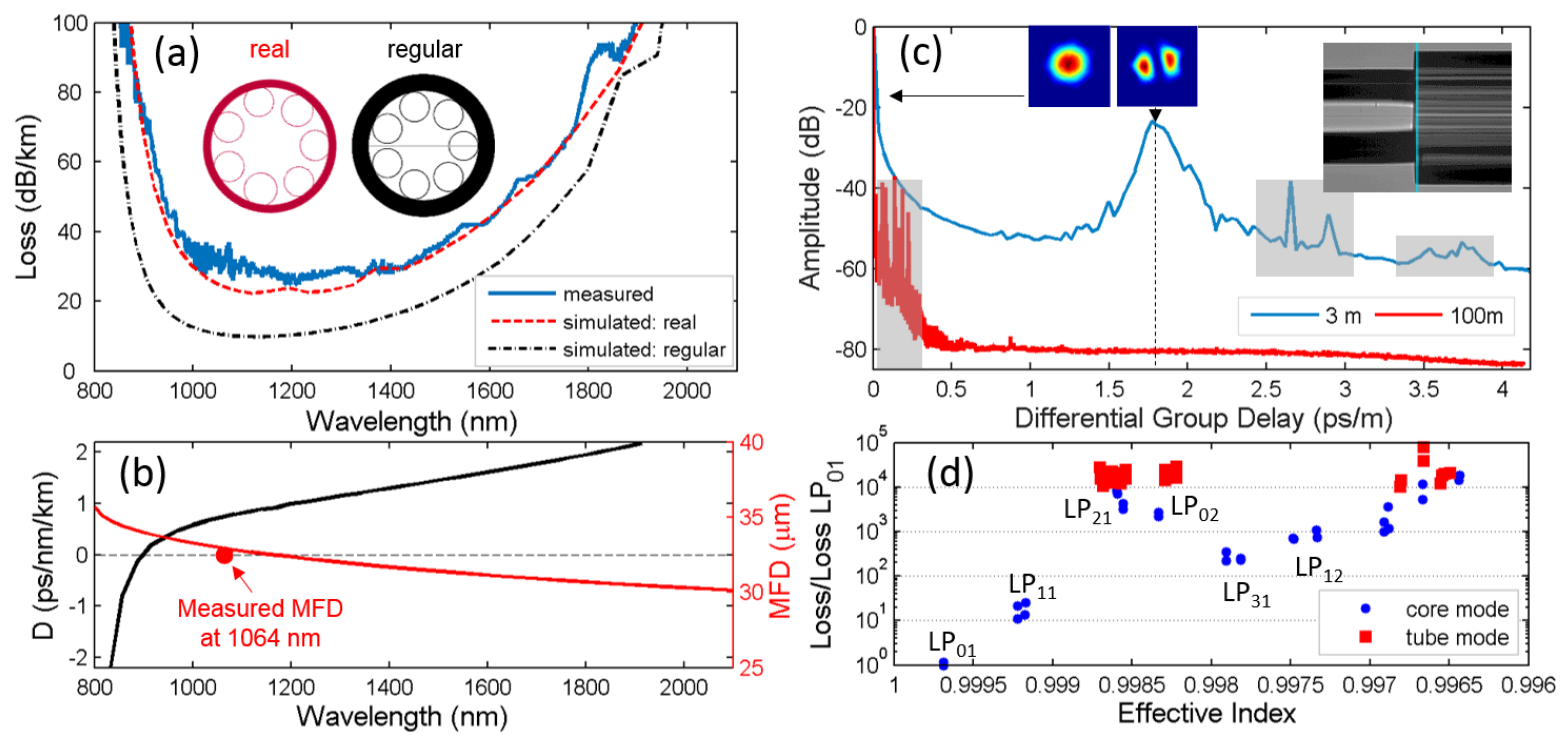

Fig.2: (a) Measured (solid) and simulated (dash) loss of the fiber in Fig.1, and simulated loss of a similar fiber with regular structure (dot-dash); (b) simulated dispersion and mode field diameter; (c) $\mathrm{S}^{2}$ measurement of the fiber with a spliced launch (inset: splice image), indicating the high modal purity obtained after $100 \mathrm{~m}$. The grayed area shows known measurement artifacts; (d) simulated differential loss of the first 50 modes.

Modelling of the actual structure with a finite element method code [11], taking into account small non-uniformities (e.g. in the inter-tube gaps), yields excellent agreement with the experimental data, as shown in Fig.2a. This confirms that the dominant loss mechanism in these fibers is leakage loss; as a result of the antiresonance operation which expels light from the membranes and produces a very small fraction of optical power in the glass $\left(1-8 \times 10^{-5}\right)$, surface scattering loss, which is prevalent in HC-PBGFs, has only a negligible contribution $(\sim 0.1 \mathrm{~dB} / \mathrm{km})$ for this fiber. Additional useful features are the very low group velocity dispersion of $<2.5 \mathrm{ps} / \mathrm{nm} / \mathrm{km}$ across the whole transmission window, and the large mode field diameter (MFD), which is almost constant at $\sim 32 \mu \mathrm{m}$ across the full octave, and which, interestingly, decreases with wavelength (Fig.2b). Despite the large MFD mismatch with SMF we have been able to achieve a fully connectorized SMF28-hollow core-SMF28 fiber with a total average connectorization loss of $\sim 2.1 \mathrm{~dB}$ (as of yet not fully optimized) through the use of suitable intermediate buffer fibers. The simulated differential mode loss (Fig.2d) indicates that the high order mode with the lowest loss (LP 11 ) is over 10 times lossier than the fundamental $\mathrm{LP}_{01}$-like mode and the loss of all other high-order modes is over 100 times greater. This is a result of resonant coupling with air modes in the cladding tubes and is a well understood effect for this type of fiber $[11,12]$. Combined with very low intermodal coupling it makes this fiber strongly single moded after just few 10 s of meters. This prediction is confirmed by the $\mathrm{S}^{2}$ measurements in Fig. $2 \mathrm{c}$ that show the absence of $\mathrm{LP}_{11}$ modes (or any other modes) after $100 \mathrm{~m}$ of fiber. This corresponds to a modal purity (high order mode 
suppression) of better than $70 \mathrm{~dB}$, the highest ever modal purity reported in a hollow core fiber to date. Simulations also indicate that simply by improving the structural regularity and by making the tubes $10 \%$ larger, a fiber with a minimum loss of $10 \mathrm{~dB} / \mathrm{km}$ and a high order mode suppression ratio of $>1000$ could be achieved (Fig.2a).

\section{Data transmission experiment}

To test the ability of the fiber to transmit data across such an enormous wavelength range we measured the data transmission performance through $100 \mathrm{~m}$ length of fiber at three widely different wavelengths: towards the short wavelength transmission edge at $1065 \mathrm{~nm}$, in the middle of the transmission band at $1565 \mathrm{~nm}$, and at the long wavelength transmission edge at $1963 \mathrm{~nm}\left(\lambda_{1-3}\right.$ in Fig.1). At the three wavelengths of interest the total span loss was 4.9, 6.3 and $18.5 \mathrm{~dB}$, respectively. A data rate of $10 \mathrm{Gbit} / \mathrm{s}$ (with a $10^{31}-1$ pseudorandom bit sequence) was used in each instance. Due to the extreme wavelength range covered it was necessary to use different combinations of lasers, modulators and detectors. In each instance the signals were passed through a polarization controller and launched into the fiber under test. At the receiver side the signal was amplified and analyzed with a bit error ratio (BER) tester. To compensate for the higher fiber loss at $1963 \mathrm{~nm}$ a Thulium-doped fiber amplifier was used. In all cases the transmission was essentially BER power penalty-free (Fig.3) confirming the excellent transmission characteristics of the fiber at these widely disparate wavelengths. Whilst we have only tested at 3 discrete wavelengths we fully expect the fiber to transmit data equally well across its entire low loss transmission window.

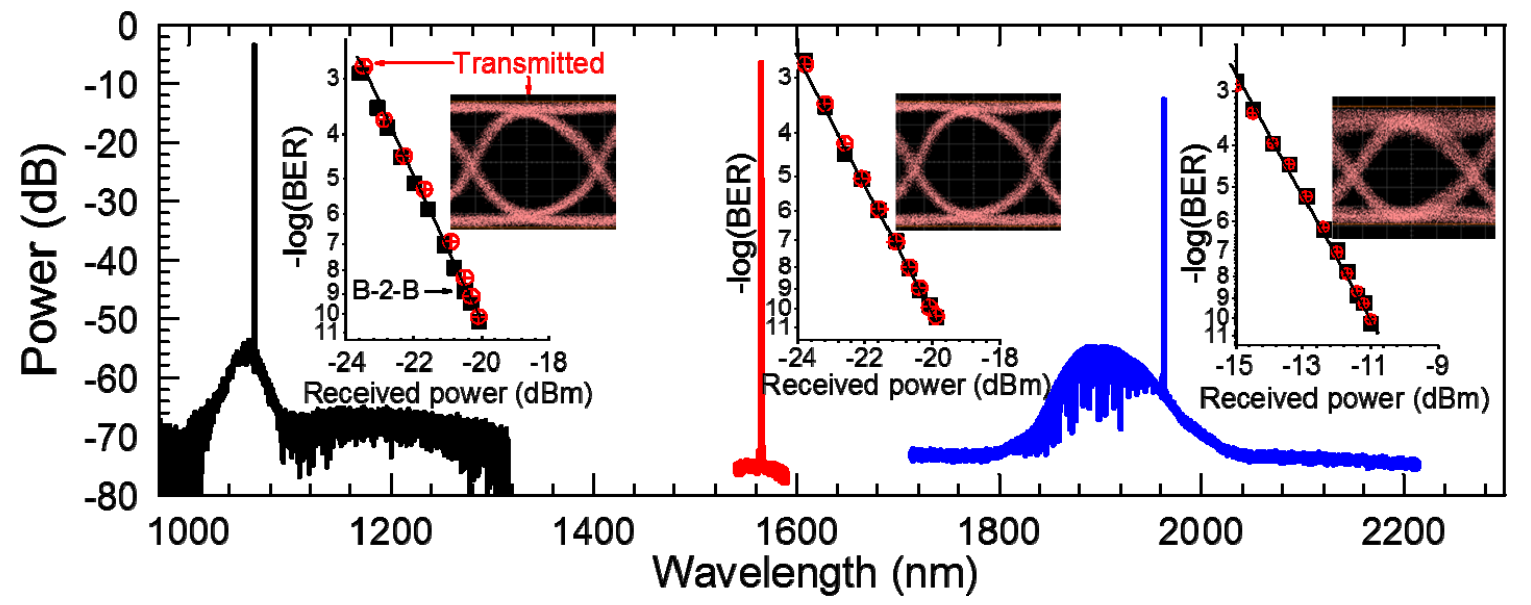

Fig.3 Result of data transmission at $10 \mathrm{Gbit} / \mathrm{s}$ at $1065 \mathrm{~nm}$ (black), $1565 \mathrm{~nm}$ (red), and $1963 \mathrm{~nm}$ (blue) wavelengths. Spectra taken with a $0.1 \mathrm{~nm}$ resolution. The insets show the BER curves (back-to-back: black squares; after transmission: red circles) and eye diagrams after the transmission.

\section{Conclusions}

We have reported the first hollow core anti-resonant fiber capable of data transmission at wavelengths separated by nearly one octave. The present fiber has shown penalty-free transmission at 1065, 1565 and 1963 nm wavelengths, but future versions could simultaneously cover the perhaps even more attractive wavelengths of 850,1300 and 1550 $\mathrm{nm}$, where the fiber's low latency, dispersion and nonlinearity would be extremely attractive features for short reach communications. Despite its large MFD, after $100 \mathrm{~m}$ the fiber was measured to have the purest single mode behavior ever reported in a hollow core fiber, and fully spliced connections with SMF28 were achieved at modest additional loss $(\sim 1 \mathrm{~dB}$ per connection, with further reduction anticipated). Loss reduction from the reported 25 $\mathrm{dB} / \mathrm{km}$ down to $\sim 10 \mathrm{~dB} / \mathrm{km}$ level should be possible through realistic improvements of the fiber structure, making $\mathrm{km}$-scale reaches possible, e.g. for intra data center applications. Finally, simulations indicate that through the inclusion of more complicated tubular structures (specifically inner nested tubes), the loss of these fibers is predicted to drop by two further orders of magnitude or more [11], making these antiresonant hollow core fibers one of the most promising novel fiber technologies currently under investigation.

Acknowledgements. This work was partly funded by the UK EPSRC through grant EP/I061196X

\section{References}

[1] F. Poletti, et al., Nature Photonics 7, 279-284 (2013).

[2] Y. Chen, et al., J. Lightwave Technol. 34(1), 104-113, (2016).

[3] M. Kuschnerov et al., Proc. ECOC 2015, paper Th.1.2.4

[4] B.J. Mangan, et al., Proc OFC 2004, paper PD24.

[5] N.V. Wheeler, et al., Proc OFC 2012, paper PDP5A.2.

[6] V.M. Sleiffer, et al., J. Lightwave Technol. 32(4), 854-863 (2014).
[7] B. Debord et al., Opt. Express, 21(23), 28597-28608 (2013).

[8] F. Yu et al., Opt. Express, 20(10), 11153-11158(2012)

[9] A. N. Kolyadin et al., Opt. Express 21(8), 9514-9519 (2013).

[10] P. Uebel et al., in Proc Frontiers in Optics 2015, paper FW6C.2

[11] F. Poletti, Opt. Express, 22(20), 23807-23828 (2014).

[12] L. Vincetti et al., Opt. Express, 18(22), 23133-23146 (2010). 